\title{
System Architecture for Blockchain Based Transparency of Supply Chain Social Sustainability
}

Accepted by Robotics and Computer-Integrated Manufacturing in November 2019

\author{
V G Venkatesh ${ }^{\mathrm{a}}$, Kai Kang ${ }^{\mathrm{b}}$, Bill Wang ${ }^{\mathrm{c}}$, Ray Y Zhong ${ }^{\mathrm{b}}$, Abraham Zhang (corresponding \\ author, E-mail: abraham.zhang@ aut.ac.nz $)^{\text {c,d }}$ \\ ${ }^{a}$ EM Normandie Business School, Métis Lab, 30 Rue de Richelieu, 76087 Le Havre, France. \\ ${ }^{b}$ Department of Industrial and Manufacturing Systems Engineering, The University of Hong \\ Kong, Hong Kong \\ ${ }^{c}$ Auckland University of Technology (AUT) Business School, AUT University, Private Bag \\ 92006, Auckland 1142, New Zealand \\ ${ }^{\mathrm{d}}$ Lumen Research Institute, Excelsia College and Indiana Wesleyan University, 69-71 \\ Waterloo Road, Macquarie Park, NSW 2113 Australia
}

\begin{abstract}
Social sustainability is a major concern in global supply chains for protecting workers from exploitation and for providing a safe working environment. Although there are stipulated standards to govern supply chain social sustainability, it is not uncommon to hear of businesses being reported for noncompliance issues. Even reputable firms such as Unilever have been criticized for production labor exploitation. Consumers now increasingly expect sellers to disclose information on social sustainability, but sellers are confronted with the challenge of traceability in their multi-tier global supply chains. Blockchain offers a promising future to achieve instant traceability in supply chain social sustainability. This study develops a system architecture that integrates the use of blockchain, internet-of-things (IoT) and big data analytics to allow sellers to monitor their supply chain social sustainability efficiently and effectively. System implementation cost and potential challenges are analyzed before the research is concluded.
\end{abstract}

Keywords: Blockchain; social sustainability; multi-tier supply chain; supply chain sustainability; traceability.

Article Classification: Research Article 


\section{Introduction}

Global Supply Chains (GSCs) are becoming increasingly complex over the years. Consequently, it has become more challenging to manage social sustainability issues which are concerned by many overseas buyers (including large retailers and brand owners) and consumers. Social sustainability in GSCs deals with matters related with providing suitable working conditions by protecting workers from exploitation, maintaining healthy and safe enviornment with fair wages and equal treatment, offering employee training, and encouraging freedom of association [1-3]. The standard of supply chain social sustainability is mainly controlled by buyers or third-party proposed norms, otherwise known as supplier code of conduct (SCC), which offer guidelines to suppliers [2, 4]. However, there is a growing concern around SCC implementation issues, which need close monitoring and follow-up audits, especially after major industry incidents including the Unilever labor exploitation scandal, the Rana Plaza building collapse, the Foxconn employee suicides, and the Karachi fire accidents $[5,6]$. Such incidents not only damage brand reputation, but raise questions around social sustainability compliance governance and its monitoring mechanisms across various supply chain layers.

In the above context, SCC implementation is becoming strategically important and it requires commitments from multiple functions including human resource management, training, sourcing, and supply chain management [7, 8]. It involves complex internal documentation and external audit and certification reports that are traditionally kept confidential in the social sustainability compliance arena and away from the public domain $[9,10]$. For example, some labor-intensive product sectors, like toys and apparel sectors, often use undeclared suppliers in developing countries which makes it a challenge to ensure social sustainability compliance across multi-tier supply chains. Multiple stakeholders, including brand owners, retailers, and non-governmental organizations (NGOs), struggle to monitor and control social sustainability problems in global supply chains [11,12]. Also, pressing customer requirements and intricacies in managing global production networks demand a high level of operational flexibility [13]. In addition, this is further complicated by the growth of ubiquitous manufacturing strategies, wherein design, manufacturing, and sales are undertaken by different stakeholders by sharing the pool of configurable resources [14]. Hence, there is a greater need to improve supply chain transparency, traceability, and information exchanges among stakeholders in order to manage social sustainability more effectively so as to improve compliance and to build greater trust $[15,16]$. In recent years, there is a growing interest in using smart technologies that help supply chains to be oriented towards long-term sustainability goals [13, 17].

Conventionally, supply chains depend on centralized management systems such as enterprise resource planning systems for managing the information flow of supply chains. Such centralized systems are vulnerable to error, hacking, and corruption problems. Blockchain 
technology, which is an emerging smart technology, can manage these issues effectively. It is a digital, decentralized, and disruptive innovation, in which transactions are recorded in chronological order with the objective of creating permanent and tamper-proof records [18]. It may facilitate transparency, security, durability and process integrity in supply chains [19]. It also involves global level transactions, process disintermediation, and decentralization amongst various stakeholders to provide timely services and goods [20]. Furthermore, the technology supports smart contract governance to enable supply chain actors to automate their certification contracts and processes.

The application of blockchain technology for environmental sustainability is a topical research area [19] as it can help overcome barriers to green supply chain management [21]. However, there is very limited attention on the use of this technology for managing supply chain social sustainability. Social sustainability management is complex and faces unique challenges. For example, SCC requires manufacturer suppliers to keep an honest record of data on the safety and wellbeing of their employees. However, when buyers and their auditing agencies identify potential issues in suppliers' records, such as wage and leave data, suppliers often manipulate data to cover up noncompliance. Blockchain technology, being used together with Internet of Things (IoT) and big data analytics, can thwart such opportunistic behaviors by automating data collection and recording updates, and by building tamper-proof record blocks that prevent data manipulation.

Despite the interest in supply chain social responsibility research [22-24] and the emerging attempts to apply blockchain in practice [25], knowledge of blockchain applications in supply chain social sustainability remains limited. This research aims to narrow the knowledge gap by developing a system architecture that integrates the blockchain technology, IoT and big data analytics for traceability in supply chain social sustainability. The research makes significant contributions by introducing blockchain technology into supply chain social sustainability management (SCSSM). This pioneering work opens a new research field and enriches both SCSSM and blockchain technology literature. In addition, the conceptual work necessitates a recognition of the differentiated and delineated opportunities specific to social sustainability, and it serves to broaden managerial understanding of the blockchain technology application.

The remainder of the paper is organized as follows. Section 2 reviews the relevant literature on blockchain technology. Section 3 describes the research problem of supply chain social sustainability. Section 4 develops a system architecture that integrates the use of blockchain, IoT and big data analytics for social sustainability traceability. Section 5 conducts a preliminary analysis of the cost and potential challenges of implementing such a system. Section 6 concludes the work, addresses implications for research and practice, outlines the limitations of this study, and proposes possible avenues for future research. 


\section{Literature Review}

A blockchain is a public, distributed ledger that can record transactions between any two parties efficiently in a verifiable and permanent way on a global basis [26]. This concept was proposed in 2009 by Satoshi Nakamoto along with Bitcoin [27]. In a blockchain system, each block is identified by its cryptographic hash, and connected with others, based on the hash of the previous block to form a chain of blocks [28].

Blockchain technology reduces the role of intermediaries whose servers are vulnerable to crashing, fraud, and hacks [29]. It is primarily a distributed ledger system that maintains the integrity of data transactions and enhances the traceability of production and logistics activities. When applying blockchain technology, trust is embedded in and programmed into the technological platform [25]. This has facilitated the creation and movement of digital assets. It also encompasses a smart contract module which stores negotiated terms and verifies the outcomes against the agreed conditions. This serves to increase transparency along supply chains by reducing the role of intermediaries [19]. Moreover, the system transactions are continuously verified, authorized, and stored by the digital blocks and connected with the preceding ones, forming a chain which facilitates material and information transparency. Eventually, supply chains begin to focus on knowledge and communication components, rather than material characteristics. However, the traceability and transparency enabled by blockchain may shift the balance of power in traditional governance structures [30]. In some cases, it also provides a direct or indirect control on firm investments, as the system handles financial data (including debt and liquidity status) transparently across stakeholders.

The decentralization attribute of blockchain facilitates the distribution of the same information across the whole network as no single node can control the transaction. The structure has permanent time stamps and prevents any possible attempts of information modification or adulteration, without the approval of all stakeholders, thus, driving a collective responsibility to ensure data safety and reliability [27,31]. In some cases, it can be customized through special permissions by converting them as private ledgers. More precisely, the transaction security is enhanced through the digital signatures and asymmetric cryptographic algorithm, with both public and private keys. Each stakeholder performs a transaction, for example, an asset sale or modifying the records, by using a private key and which is further validated using public keys [27]. It also reduces transactions costs such as the cost of verifying the attributes of a transaction and exchange cost, without relying on intermediaries. Firms are looking at this technology as a democratizing tool in the future IoT [32]. Typical inter-organizational barriers can be removed through blockchain technology deployment, as it provides a smart contract environment with an immutable public ledger to view every transaction and supports independent process monitoring from a global perspective. However, some organizations are 
hesitant to be more transparent under this distributed ledger system, which may limit the full benefits of blockchain technology [19].

Table 1 - Blockchain vs centralized systems

\begin{tabular}{|c|c|c|}
\hline & Blockchain based systems & Existing centralized systems \\
\hline Access & $\begin{array}{l}\text { Open cum closed environments } \\
\text { and decentralized process. }\end{array}$ & $\begin{array}{l}\text { It is primarily a restricted and closed } \\
\text { transaction environment. }\end{array}$ \\
\hline Control & Multi-stakeholder control. & Single authority control. \\
\hline Security & $\begin{array}{l}\text { High security - less probability } \\
\text { for information tweaking and } \\
\text { filtering. All stakeholders will } \\
\text { have the same information at any } \\
\text { point in time. }\end{array}$ & $\begin{array}{l}\text { Chances of tampering and misleading } \\
\text { information - prone to information } \\
\text { damage and filtering at all possible } \\
\text { levels. Stakeholders will have similar } \\
\text { information only if permitted by the } \\
\text { central authority. }\end{array}$ \\
\hline $\begin{array}{l}\text { System } \\
\text { update }\end{array}$ & $\begin{array}{l}\text { Instant update of all supply chain } \\
\text { events. }\end{array}$ & $\begin{array}{l}\text { It may have some update delays due to } \\
\text { the control of a single authority. }\end{array}$ \\
\hline $\begin{array}{l}\text { Governance } \\
\text { structure }\end{array}$ & $\begin{array}{l}\text { The governance structure is } \\
\text { transparent, with clear roles and } \\
\text { responsibilities. }\end{array}$ & $\begin{array}{l}\text { Although it appears to establish clear } \\
\text { roles and responsibilities, governance } \\
\text { is highly dependent on the power } \\
\text { dynamics with a single authority. }\end{array}$ \\
\hline $\begin{array}{l}\text { Information } \\
\text { reliability }\end{array}$ & $\begin{array}{l}\text { High information reliability, as it } \\
\text { directly comes from the } \\
\text { respective stakeholders. Fewer } \\
\text { rounds of further validation. }\end{array}$ & $\begin{array}{l}\text { Compared to the blockchain } \\
\text { environment, information reliability is } \\
\text { low as data can easily be filtered. It } \\
\text { normally invites further rounds of } \\
\text { validation. }\end{array}$ \\
\hline $\begin{array}{l}\text { System } \\
\text { integration }\end{array}$ & $\begin{array}{l}\text { System integration is a common } \\
\text { and base event. }\end{array}$ & $\begin{array}{l}\text { Information integration and } \\
\text { synchronization is a separate activity, } \\
\text { as external stakeholders need to align } \\
\text { with the sponsor. }\end{array}$ \\
\hline
\end{tabular}

Source: $[33,34,35]$

Table 1 compares the blockchain based systems with the existing centralized systems. The key unique attributes and claimed advantages of blockchain have elicited strong interest from practitioners and researchers in this disruptive technology since 2015 [18]. In financial services, blockchain enables all financial transactions, including cryptocurrencies, since the technology is mainly used to legitimize the currency [36]. In healthcare, the technology supports the exchange of health records electronically and ensures adherence to privacy concerns. This is possible through a specific healthcare data gateway (HDG) for data control 
[37]. Moreover, the disruptive technology helps to trace the carbon footprint of products and to track substandard outputs, both of which directly and indirectly facilitate resource consumption planning and the control of greenhouse gas emissions [19].

Since blockchain can be regarded as an application layer operating on the top of internet protocols that enable economic transactions between business actors without relying on a third party [25], many researchers have tried to explore the impact of blockchain technology on supply chain management [18, 19], including supply chain coordination [38]. In addition, potential applications of blockchain have been explored to enhance the sustainability of the supply chain, since this technology can store data that need to be identified and verified. For instance, an agri-food supply chain traceability system has been developed to guarantee food safety, based on RFID and blockchain technology [37]. Blockchain can bring supply chain transparency to a new level and expose negative practices such as child labor upstream in the manufacturing process and the unethical use of rainforest resources [37]. Saberi et al. [19] investigate activities across and within the supply chain and then provide valuable insights into the diversified sustainability-oriented opportunities associated with blockchain technology.

\section{Social Sustainability in Global Supply Chains}

Social sustainability in supply chains which focus on the health and well-being of the people involved in the supply chain, has gained attention that is separate to concerns of environmental sustainability. Brands like Walmart have the best practices in maintaining environmental standards but are often criticized for gaps in meeting social sustainability requirements [39]. Reputable brands are often criticized for their production lines in exploitative working conditions, especially in offshore locations where the prime objective is achieving low costs. Recent incidents from developing nations such as Bangladesh, Pakistan, and India, in conjunction with an increased awareness and sensitivity on the part of customers in the global supply chains have compelled stakeholders to uphold social sustainability norms, which is one of the key expectations of GSCs [5, 40].

Fig.1 depicts the characteristics of social sustainability practices adopted in global supply chains and its problems. In general, sustainability practices are guided by SCC, or by supplier sustainability norms, as an extension to sourcing contracts [41-43]. They are the tools of the buying organizations to improve responsible practices of any GSC and provide a means of effectively maintaining their own image in the market $[1,44]$. Many of these standards have evolved only in recent times as strategic elements of any world-class sustainable framework [11]. They provide guidelines on how to manage child labor, occupational health and safety, legal minimum wage, the working environment, training and freedom of association, and reducing the exploitation of workers by regulating their working hours and insurance [7, 40, 45-47]. Although these norms are not always regulated by local legislation, they address some of the major challenges of globalization by enforcing basic human rights on stakeholders [48- 
50]. Subsequently, social sustainability governance of a GSC has become a mainstream practice of brands/buyers. It demands certifications, which involves documentation reviews and factory floor audits for health and safety requirements either through their own representatives or through third-party agencies.

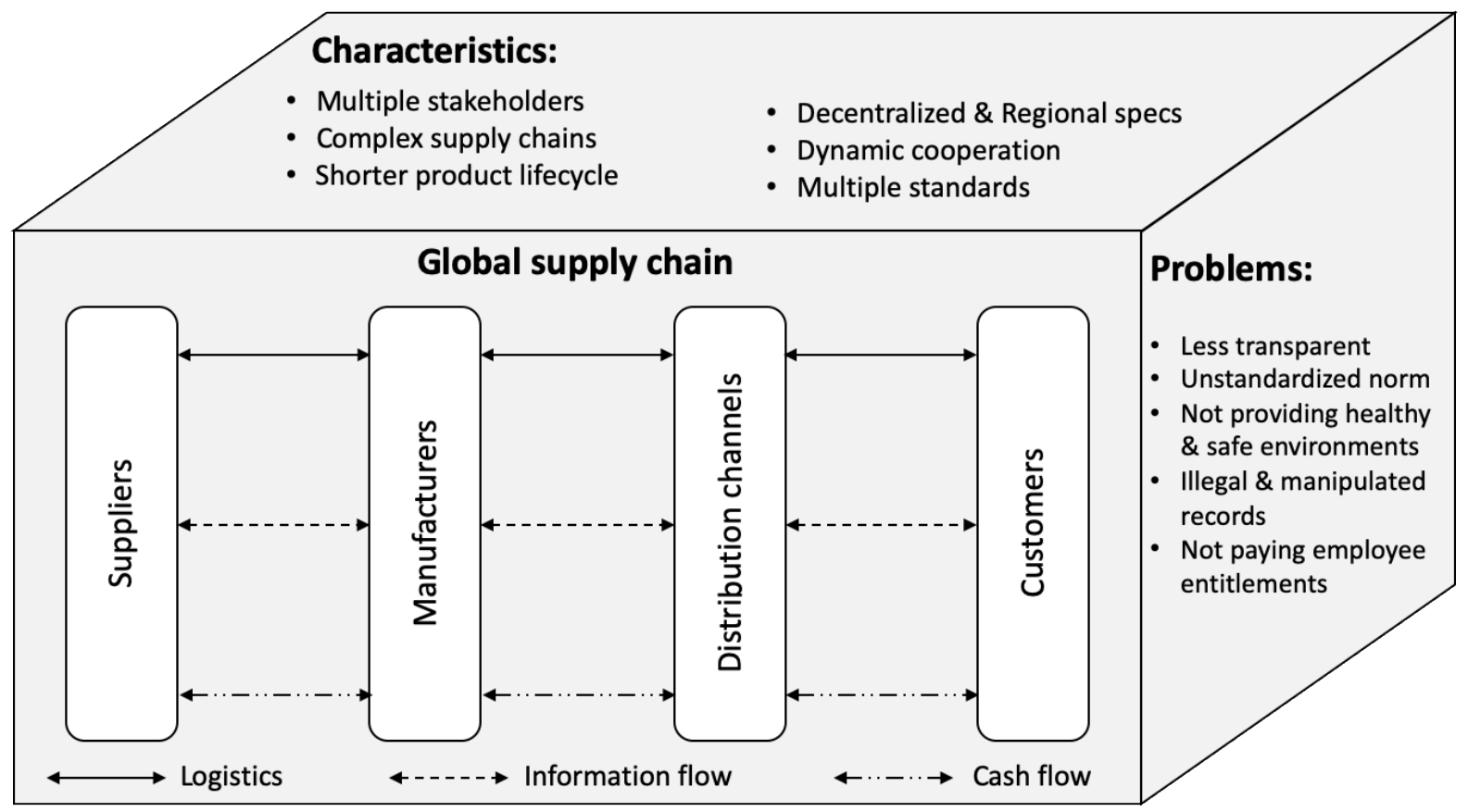

Figure 1 Social sustainability attributes of global supply chains

Global supply chains are complex and need to manage sustainability across both upstream and downstream operations [51]. Brands like Puma and Nike demand and audit sustainable practices beyond their first-tier suppliers. This means sub-suppliers and subcontractors also need to be monitored and to comply with sustainability requirements [52]. They also highlight the importance of enhancing social sustainability capabilities in terms of skills, practices, and organizational processes in both technical and relational ways [5, 12, 53]. Technical considerations include compliance systems, and procedures and their reporting, which significantly reduce communication gaps and costs. This is facilitated through stringent control mechanisms such as certifications and incentive systems that serve to impact supplier evaluation and the monitoring of social sustainability compliance [54, 55]. However, the system suffers from multiple standards which do not always agree with each other. Thus, as the supply chain grows, it encounters many challenges in streamlining the social sustainability practices. The most important ones include less transparency, unstandardized norms in implementing the minimum wage and payment schedule, not providing a healthy and safe working environment (including the non-maintenance of facilities such as creches), illegal and manipulated over-time records, not paying employee insurance and other entitlements (like gratuities) on time. These sorts of problems eventually lead to the issue of providing the right information to external stakeholders who are keen to maintain and monitor social sustainability practices in a supply chain. 
To effectively counter the aforementioned challenges, research reports endorse the Evidence, Verifiability and Enforceability (EVE) framework, and also endorse the deployment of blockchains to monitor the sustainable supply chain performance, thereby increasing its transparency [55]. Furthermore, the system is expected to provide regular feedback on social sustainability performance along the supply chains to the focal firms and their stakeholders, which makes compliance and follow-up processes more transparent. Overall, the future holds great promise in the application of blockchain technology to enhance SCSSM, but very limited attention has been given to this research topic thus far. Therefore, this study attempts to narrow the knowledge gap by developing a system architecture for guiding the deployment of blockchain for SCSSM.

\section{System Architecture}

This section presents a system architecture for the blockchain-based supply chain social sustainability management (BSCSSM) system. The key applications and services are described as follows.

\subsection{Overview of system architecture}

Figure 2 illustrates the system architecture of BSCSSM system including five main layers: smart objects, communication channels, data analysis, blockchain network, and applications.

Smart objects form the bottom layer of the architecture. Smart objects refer to typical resources in SCSSM, including man, machine, material, method and environment (4M1E) which can be transformed into smart objects by suitable IoT devices. For example, the related data of employees ("man") including heartbeat, working hours, movements, etc can be detected and collected by wearable technologies such as smart bracelets and shoes [56]. These collected data can then be transmitted, processed, and recorded to assist decision making. This layer is mainly used to collect multisource data by various IoT devices in a timely manner, accurately and authentically.

The immediate upper layer is the communication channel layer. The data obtained from smart objects are transmitted via diversified channels, including ZigBee, Bluetooth, WiFi, Transmission Control Protocol/Internet Protocol (TCP/IP), Ultra-wideband and 5G. This layer is responsible for managing diversified communication channels in an easy-to-deploy and flexible-to-configure manner, which standardizes heterogeneous channels and guarantees the data transmission stability.

In the data analysis layer, there are four key procedures: data cleansing and format conversion, classification and aggregation, pattern excavation and interpretation, and standardization and representation [57]. Data cleansing aims to detect and delete noise data which are incomplete, inaccurate and redundant. The cleansed data is then converted to a standard format if required. 
Format conversion is necessary as multiple supply chain stakeholders use a variety of applications and encode data in different ways. Supply chain stakeholders need to agree on the standard data format to be used in the BSCSSM system for data sharing and processing. After data cleansing and format conversion, the standardized data is classified and aggregated into different specific groups, which are then further processed to mine pattern recognition from a temporal and spatial perspective. From this, the discovered patterns can be interpreted, which is beneficial to presentations in different situations and can be used for supporting various applications in the application layer. For instance, through regression methods, trajectory knowledge of the learning curves of senior, intermediate and junior logistics operators is excavated from the fitted curves in a time interval, which is pattern excavation and interpretation. The next step, standardization and representation, is used for formatting the results data through some standardized models, such as XML, which could be used for all other heterogeneous systems. Such standardized models are defined by the ISA-95 international standard.

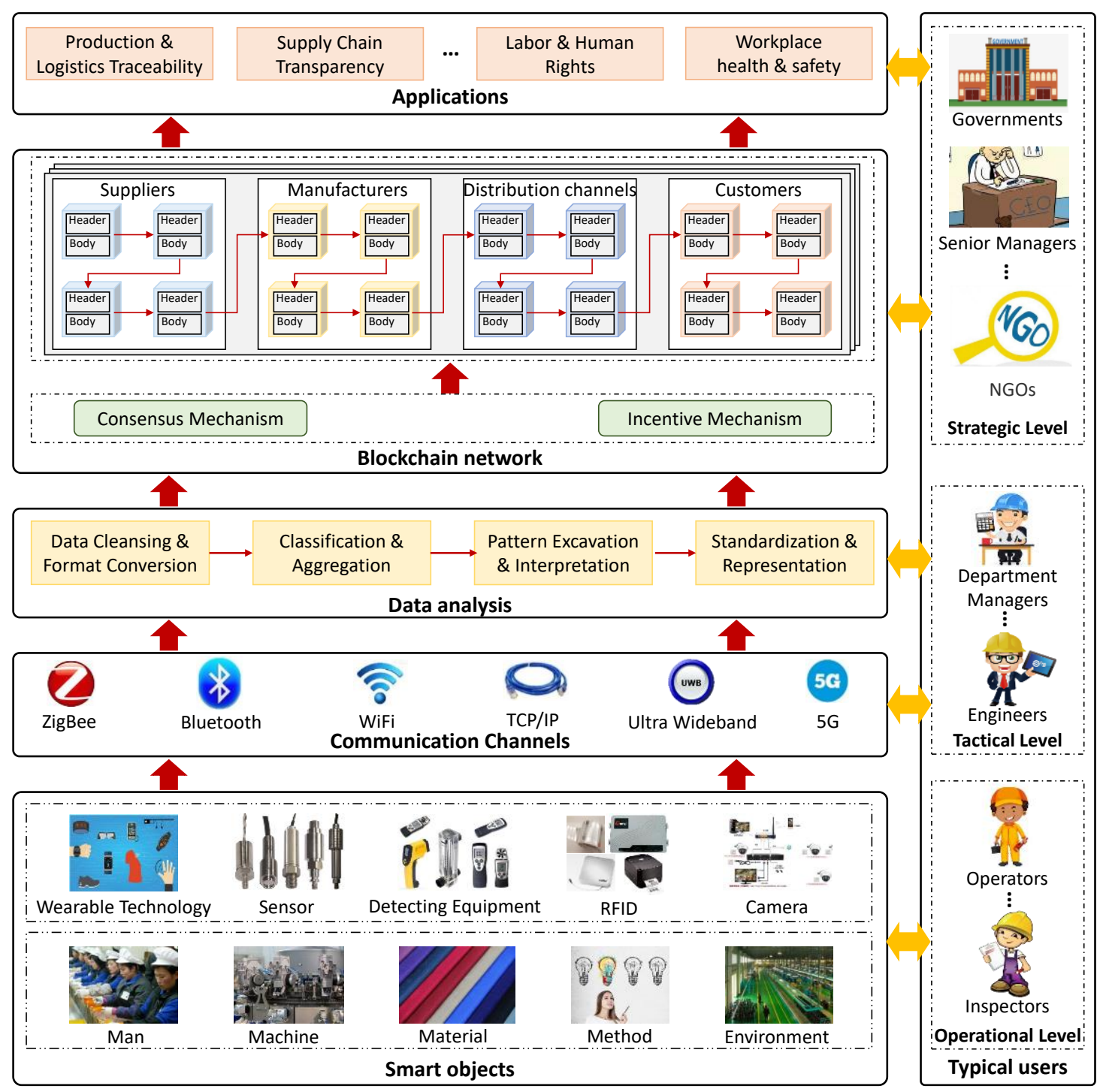


Figure 2 System architecture of blockchain-based supply chain social sustainability management system

The processed data is used for forming a series of blocks in the blockchain network layer. A block in the blockchain network contains a header and a body [58]. The header consists of a previous block hash, time stamp, merkle root, nonce and target difficulty. The body consists of key components in $4 \mathrm{M} 1 \mathrm{E}$, such as the physiology of a worker and operation status of a machine. The blocks are connected in chronological order, forming blocks and finally blockchain networks. In the forming process, a consensus mechanism is required to guarantee the data consistency and the fault-tolerant ability among distributed ledgers while an incentive mechanism is designed to motivate stakeholders to record data.

In the top layer there are various applications and services for SCSSM, including production and logistics traceability, supply chain transparency, human resource management, and workplace health and safety (see Section 3.3). These applications and services are created and enabled by the blockchain network, using processed data collected from smart objects by IoT technologies.

\subsection{Typical users}

In the system architecture there are three types of typical users using or supporting relevant layers following the widely accepted strategic, tactical, and operational level management in supply chains [59].

At the operational level, typical users can be front-line operators and inspectors. All the raw data in SCSSM originates from this level. For example, aided by the wearable technologies, the physiological status of employees can be detected and monitored and their movements recorded [56]. These types of data can be used to further analyze the workload of employees. Meanwhile, other types of data such as levels of noise and temperature readings in work floors can be monitored, which makes the working environment transparent and enables the improvement of working conditions. Moreover, operational level users need to verify the authenticity of data captured by IoT technologies. For example, workers should verify whether their own working hours captured by wearable technology are consistent with the reality.

At the tactical level, typical users, including department managers and engineers, aim to guarantee the stability of data transmission by deploying and integrating heterogeneous channels. They also need to design a variety of models/algorithms for processing the data to get insights from the data. For example, a big data approach can be used to discover the meaningful and useful patterns based on IoT-captured data, such as logistics trajectories [57] and standard operation times [60]. Moreover, typical users, like department managers, need to verify the authenticity of the data. Only after tactical and operational level users reach a 
consensus on the IoT-captured data can the data be further utilized for data analysis and transmitted to other nodes in the blockchain network.

At the strategic level, typical users can be senior managers and relevant governmental organizations and non-governmental organizations (NGOs). They are responsible for establishing the blockchain network, including the consensus mechanism and incentive mechanism design, as well as the provision of hardware. They also act as decision-makers on strategies and other policy issues using the information and data from the blockchain network. For example, senior managers of SCSSM can observe the environment and conditions of work floors, such as the room temperature, humidity and noise pollution. Governmental organizations and NGOs may also become involved in the process. Based on these data, some necessary measures are to be taken to ensure the health and safety of the employees.

\subsection{Key applications}

To achieve SCSSM, the following four key applications are possible through the above proposed blockchain architecture.

- Production and logistics traceability enable companies to identify and verify the elements and chronology of events within supply chains; it aims to offer the right information to the right person at the right time to realize optimal supply chain management [61]. Through production and logistics traceability, companies can readily trace and track the production and logistics processes, which helps companies to detect a variety of problems such as machine breakdowns and defective raw materials. Through this application, governmental organizations and NGOs can also scrutinize the social sustainability of the companies and take actions to prevent the abuse of human rights and to control the scale of any identified hazards [37]. Overall, this application helps companies to optimize their supply chain configuration, lower the cost of production and logistics, and facilitate the improvement of SCSSM.

- Supply chain transparency refers to information readily available to end-users and firms in a supply chain [62]. This application in our system architecture can make it possible for other stakeholders, such as governmental and non-governmental organizations, to access information relating to socially sustainable practices because of the attribution of the blockchain network [63]. Generally, supply chain transparency is more concerned with social sustainability in supply chains than with production and logistics traceability. Given the increasing complexities of a highly fragmented production network, retailers may find it more difficult to identify whether other supply chain actors have complied with SCC or with other standards and certificates under global sourcing circumstances [64]. This application can effectively meet the requirements of retailers, even in multi-tier supply chains which include multiple small- and medium-sized enterprises and subcontractors 
participating in production activities. The blockchain technology of this application enables all supply chain members, including retailers, to easily check whether all tiers of suppliers have obtained social sustainability certificates, are conducting satisfactory labor practices, and maintaining adequate workplace health and safety records.

- Labor and human rights focus on labor related issues in SCSSM, including reasonable working conditions, working hours, living wages, social welfare and equity, etc. These aspects are significant components of social sustainability [65]. Assurance of human rights is a basic criterion to certify the social sustainability of a company. For example, retailers, governments and NGOs can retrieve the working hours of workers from a blockchain network to judge whether companies are violating the overtime limits and whether living wages are paid to workers on time. These actions and efforts will be recorded in a blockchain network, which can be regarded as an instrument to support social sustainability certifications.

- Workplace health and safety is an application concerned with the safety, health and welfare of workers in the workplace. Lax workplace health and safety practices may lead to a reduction in work efficiency and to serious incidents and hazards [66]. A blockchain-based supply chain can establish tamper-proof records, such as whether a facility has the required fire safety and building safety certificates. Government agencies, third-party inspection agencies, and industry associations may be involved in building the blocks by issuing and authenticating the certificates. In addition, the data related to the working conditions (such as lighting, temperature, humidity, noise and ventilation) can be captured and collected by IoT technologies before they can be stored in the blockchain network. These data can be utilized to aid the management of the working environment and business performance. In addition, data relating to staff training and learning of occupational health and safety matters can be recorded in the blockchain network as basic SCSSM requirements.

\section{Discussion}

\subsection{Cost of the System}

The investment of the blockchain-based SCSSM system mainly consists of hardware costs, software costs and other costs. We provide a cost estimate based on a Chinese manufacturer which employees about 2,100 workers responsible for production and logistics activities. They operate on three shifts with 700 workers on each shift. The company runs six manufacturing lines which are equipped with about 1,000 machines plus 50 buffers. The cost of the system is calculated based on the 2018 constant price.

Hardware costs include the cost of wearable technologies, sensors/detecting equipment, RFID technologies, and the installation and maintenance cost of these hardware. Apart from computers, hardware devices are generally used to maintain reliability and readability for the 
next 18 months $[67,68]$. Smart bracelets, as one of the most mature wearable technologies, is chosen to collect data on employees. Thus, the total cost of wearable technologies is $¥ 0.7$ million/year, based on an average smart bracelet cost of $¥ 500$ each. A variety of sensors/detecting equipment are deployed to monitor the status of the working environment. If lighting, temperature, humidity, noise and ventilation are monitored, then five types of sensors/detecting equipment (e.g. illuminometer, thermometer and humidity-meter, etc.) must be deployed. Assuming that each type of sensor/detecting equipment can only monitor five machines, 1,000 sensors/detecting equipment would be required. Based on an average sensor/detecting equipment cost of $¥ 240$ each, the total cost is $¥ 0.16$ million/year. There are about 10,000 batches of products operated daily in the work floor and each batch is attached by an RFID tag tracked by about 300 RFID devices. If we factor all the cost of RFID technologies into tags, then each tag would cost about $¥ 2$ each. Therefore, 10,000 tags used to track 10,000 batches will cost $¥ 20,000$, and the total cost of the use of RFID technologies is $¥ 0.013$ million/year if RFID devices and tags are updated every 18 months. Moreover, in order to record data obtained manually and captured automatically to form blockchain networks, the cost of local server storage is added to hardware costs annually, which is about $¥ 0.5$ million. The installation and maintenance of such hardware would cost about $¥ 0.15$ million/year. According to the above cost analysis, the total cost of hardware will be $¥ 1.523$ million/year.

Software cost is used to develop the new system proposed in this paper. There are generally two approaches. The first approach is to outsource all computer software tasks, including design, development, maintenance, testing and evaluation, but it is hard to estimate the cost because different software providers may quote very different prices for the development of this system. The other approach is for the company to complete all tasks by itself, which requires the employment of between 5 to 8 software engineers. According to a survey published in the last two years, the average salary of a software engineer in China is $¥ 8,381 /$ month [69]. If the company employs 7 software engineers responsible for development and maintenance of this system, then the total software cost can be converted into the labor cost, which would cost about $¥ 0.704$ million/year. In addition to employee salaries, other expenditures such as insurance expenses in China make it prudent for employers to budget $40 \%$ extra in expenses. Therefore, the total software cost is about $¥ 0.986$ million/year.

Other costs mainly include training costs and the cost of system breakdowns due to the switch to a BSCSSM system. Training costs consist of trainer fees and the time cost of trainees. Technicians of hardware suppliers hired to teach employees how to use the system and the associated IoT devices would cost $¥ 0.05$ million. Based on an average labor cost of $¥ 3,000$ /month for production workers, the time cost of trainees is calculated based on their wages of $¥ 1.575$ million, assuming that there is one-week training at the start of the system implementation. Thus, the total training cost is $¥ 0.163$ million/year if the cost can be amortized 
over ten years. Moreover, in the early stages of adopting a new system, it is common for businesses to suffer from system breakdowns which have adverse effects on operational productivity and customer services. A one-time cost such as this is estimated to be $¥ 0.5$ million and equivalent to $¥ 0.05$ million/year if this cost is amortized over 10 years. Therefore, the total of the other costs is $¥ 0.213$ million/year. If the hardware cost, software cost and other costs are added up, the total cost of this system is $¥ 2.722$ million/year.

\subsection{Potential Issues/Challenges and Solutions}

The proposed BSCSSM system is a complex application. Its implementation in practice will face technical issues as well as non-technical challenges with respect to how the involved stakeholders effectively collaborate. The researchers recommend some possible solutions to tackle the potential issues/challenges.

- Deployment of IoT technology is a barrier to transform physical resources into smart objects, consequently a blockchain-based SCSSM system cannot be achieved because of high costs, high risks and high levels of specialist technical skills required when adopting IoT technology [70]. Product service systems (PSS) is one of the most successful business models to deal with this problem [68]. In this model, a product is combined with its associated services and sold to customers at a reasonable price, which has been successfully applied in many fields, like aircraft engines, photocopiers and especially RFID technology [71]. User companies can choose product-, use- or result-oriented services according to their requirements [72]. For example, companies can deploy IoT devices through a sharing approach with others in their alliances, which means companies adopt use-oriented services to mitigate the problem of high cost. The relationships can be vertical partnerships existing in supply chains or a lateral association with others located in an industrial park.

- An incentive mechanism is a critical component for the construction of a blockchain network. Without an incentive mechanism, few participants would record the required data, leading to failure to build a blockchain network. Unlike cryptocurrencies, such as Bitcoin, there is not a certain number of cryptocurrencies being issued as rewards and allocated to nodes in a blockchain network [73]. Therefore, an effective incentive mechanism needs to be designed to maximize the social welfare of all nodes. A possible solution is that those who are nodes in the blockchain network should be authorized to have access rights to obtain related data, based on their access level and roles. Through authorizing access rights, stakeholders may be more willing to become one of the nodes in the blockchain network. For example, governments and NGOs can monitor all the involved businesses effectively at real time and stop them from inappropriate behaviors in time, and retailers can immediately verify the social sustainability certificates of their suppliers. 
- Worker cooperation may be a potential challenge in the deployment of some IoT devices. Although the new system is designed to protect the health and safety of workers, it may not be fully understood or supported by all workers. Some workers may feel uncomfortable with wearable devices or feel distrusted that they are being closely monitored or watched during their work, which may lead to a reluctance to cooperate with their employers. In addition, the system can potentially be used to collect and record data which infringes on the well-being of workers $[74,75]$, and it will be a challenge to avoid the "dark side" associated with blockchain technology [76]. Consequently, a non-cooperative attitude may not only increase administrative and maintenance costs, but also reduce the overall effectiveness of the system. To overcome these challenges, a technology provider must make deliberate efforts in the implementation stage to educate workers on the benefits of the system to gain the support of workers. It must also build safeguard mechanisms in the system to prevent the system from being misused.

- System implementation cost. Some shareholders may be reluctant to support an implementation of the system due to cost concerns. Although the system can strengthen a firm's sustainability image and marketing position in the long run, it does not directly improve business performance. In fact, it will reduce the return on assets in the short term. To overcome this challenge, the top management must be determined to invest in the system for sustainability and long-term benefits and align the thinking of the whole organization through regular company-wide communications.

- Government policy formulation. The Blockchain technology is disruptive and its application in businesses is still in its infancy [77]. The government faces challenges in regulating the use of the blockchain technology as it is new and still rapidly evolving. Given such a high level of uncertainty, the government needs to give careful consideration to a large variety of potential applications of this technology in multiple aspects, including security and legitimacy [78]. Furthermore, as the system involves international environments, the diversity and variations in different countries' policies and regulations must also be considered [78]. The researchers recommend that governments engage NGOs to widely consult all potential stakeholders to understand their concerns [79]. This will ensure reasonable and effective policies being drafted to regulate and guide the use of the blockchain technology.

\section{Conclusions}

This study explores how blockchain technology can potentially influence supply chain social sustainability research and practice. It develops a system architecture to integrate and apply blockchain technology, IoT, and big data analytics for social sustainability traceability in supply chains. Furthermore, it conducts a preliminary analysis of the cost and challenges of implementing the system. Overall, our research provides guidance for deploying blockchain technology to enhance traceability in supply chain social responsibility. 


\subsection{Theoretical contributions}

Our research is a pioneering work in exploring how blockchain technology can be introduced and applied to supply chains to improve monitoring and traceability in social sustainability. As an emerging disruptive technology, blockchain has incomparable merits including transparency, authenticity, trust, and security [25]. Blockchain technology has been applied in a range of domains including healthcare, financial services, and environmental sustainability $[66,80]$. While some recent publications have explored the potential influence of blockchain on supply chain management [25], there is still limited research extending blockchain to supply chain social sustainability. This research makes a significant attempt to integrate blockchain technology in supply chain social sustainability management, which enriches literature in both disciplines.

Specifically, our research proposes a system architecture integrating blockchain, IoT, and big data analytics to monitor social sustainability compliance efficiently and effectively. As chain actors in GSCs are dispersed [81] in different societies, geographic areas, industries and cultures, they often adopt different CSR standards, norms, policies, and SCCs. Consequently, traceability becomes a big challenge for SCSSM. The system architecture proposed in this study can monitor multi-tiered supply chains, to achieve instant traceability in the supply chain networks. Therefore, our study opens up a new avenue for supply chain social sustainability research.

\subsection{Managerial and policy implications}

The research provides valuable insights to supply chain practitioners. It prompts stakeholders to give serious consideration to adopting blockchain and achieve instant traceability in any multi-tier supply chain more efficiently and effectively. The proposed system offers several obvious benefits. First, it reduces the incidences of record duplication and prevents record manipulation. Blockchain technology makes it possible to easily trace the compliance history of individual suppliers. It also provides a high level of security to sensitive documents, including audit feedback and corrective action plans (CAP) which tend to be manipulated.

Second, it provides a platform to schedule social sustainability audits in a transparent manner to all stakeholders. This is useful as it coordinates all stakeholders to follow a uniform audit schedule. It can also release inspection alerts at the right time. In some cases, suppliers and other stakeholders manipulate the schedule to their advantage. This is observed especially in semi-unannounced audit environments, where buyers assign a preferred inspection time window within which audits need to be conducted.

Third, the proposed architecture supports the institutional role of governments in improving social sustainability, as some of the emerging economies like India and Bangladesh are moving 
towards an integrated and widely accepted compliance system. Hence, the work provides a direction for governing the blockchain environment by integrating all possible stakeholders under a single framework to establish a sustainable production ecosystem.

Fourth, blockchain development directly or indirectly prevents bribes and miscellaneous indirect overheads as it streamlines the social sustainability management system. In some cases, audit expenses, including service fees, are highly inflated by suppliers. The proposed system can prevent such opportunistic behavior and control expenditures in its transparency.

Lastly, the deployment of blockchain technology will serve to reduce the overall turnaround time of audits because processes of information gathering will be more streamlined and transparent. In addition, the deployment of blockchain may substantially improve intra-and inter-departmental communication as it supports integration among various departments, which will significantly reduce organizational conflicts.

\subsection{Limitations and future research}

Our research aims to explore how to apply blockchain technology into SCSSM to achieve instant traceability in supply chain social sustainability. Although the research has achieved significant results by developing a system architecture for monitoring social sustainability compliances issues, the research has several limitations. Firstly, the research on social sustainability mainly focuses on human related issues such as health and safety, labor and human rights, and transparency in procurement only. Social sustainability covers a variety of other issues including animal welfare, communities, fair trade, etc. [22]. These dimensions warrant more consideration. For example, fair trade involves a variety of stakeholders in supply chains including not only the suppliers, buyers, and other firms in the supply chain, but also other stakeholders, including governmental organizations and NGOs. Meanwhile, the understanding and acceptance of fair trade is related to many factors: the balance of long-term strategies and short-term returns and goals of supply chain actors, their positions, and levels of power in the supply chains.

There are several directions for future research. First, it would be interesting to explore how the blockchain technology influences social sustainability challenges, and particularly, the legal and ethical implications of its adoption. Second, a comparative study on blockchain enablers and barriers in different cultures and firm sizes, for example, in small-medium enterprises and in large corporations, is recommended to provide strategic insights into the implementation dynamics. Third, studies with longitudinal data are suggested to deepen our understanding on technology adoption. Fourth, the proposed system architecture needs an empirical validation. This may initially start with pilot tests in small-scale supply chains, before the system architecture is fine-tuned for large-scale implementations in a range of industries. 


\section{References}

[1] Jiang, B. (2009). The effects of interorganizational governance on supplier's compliance with SCC: An empirical examination of compliant and non-compliant suppliers. Journal of Operations Management, 27(4), 267-280.

[2] Perry, P., \& Towers, N. (2013). Conceptual framework development: CSR implementation in fashion supply chains. International Journal of Physical Distribution \& Logistics Management, 43(5/6), 478-501.

[3] Shelton, R. K., \& Wachter, K. (2005). Effects of global sourcing on textiles and apparel. Journal of Fashion Marketing and Management: An International Journal, 9(3), 318-329.

[4] Huq, F. A., Chowdhury, I. N., \& Klassen, R. D. (2016). Social management capabilities of multinational buying firms and their emerging market suppliers: An exploratory study of the clothing industry. Journal of Operations Management, 46 (September), 19-37.

[5] Huq, F. A., \& Stevenson, M. (2018). Implementing socially sustainable practices in challenging institutional contexts: Building theory from seven developing country supplier cases. Journal of Business Ethics, 1-28. https://doi.org/10.1007/s10551-018-3951-X

[6] Barber, B. (2017, May 30). Unilever falls short on labor rights. HuffPost. Retrieved from https://www.huffpost.com/entry/unilever-falls-short-on-labor-rights b 592e2089e4b047e77e4c3f89

[7] Mamic, I. (2017). Implementing codes of conduct: How businesses manage social performance in global supply chains. London: Routledge.

[8] Van Tulder, R., Van Wijk, J., \& Kolk, A. (2009). From chain liability to chain responsibility. Journal of Business Ethics, 85(2), 399-412.

[9] Bain C., Hatanaka M. (2010) The Practice of Third-Party Certification: Enhancing Environmental Sustainability and Social Justice in the Global South?. In: Higgins V., Larner W. (eds) Calculating the Social. London, UK: Palgrave Macmillan.

[10] Mitchell, R., Petra, M. A. H. Y., \& Gahan, P. (2014). The evolution of labour law in India: an overview and commentary on regulatory objectives and development. Asian Journal of Law and Society, 1(2), 413-453.

[11] Klassen, R. D., \& Vereecke, A. (2012). Social issues in supply chains: Capabilities link responsibility, risk (opportunity), and performance. International Journal of Production Economics, 140(1), 103-115.

[12] Parmigiani, A., \& Rivera-Santos, M. (2011). Clearing a path through the forest: A meta-review of interorganizational relationships. Journal of Management, 37(4), 1108-1136.

[13] Ding, K., Jiang, P., \& Su, S. (2018). RFID-enabled social manufacturing system for inter-enterprise monitoring and dispatching of integrated production and transportation tasks. Robotics and ComputerIntegrated Manufacturing, 49 (February), 120-133.

[14] Chen, T., \& Tsai, H. R. (2017). Ubiquitous manufacturing: Current practices, challenges, and opportunities. Robotics and Computer-Integrated Manufacturing, 45 (June), 126-132.

[15] Zorzini, M., Hendry, L. C., Huq, F. A., \& Stevenson, M. (2015). Socially responsible sourcing: Reviewing the literature and its use of theory. International Journal of Operations \& Production Management, 35(1), 60-109.

[16] Grimm, J. H., Hofstetter, J. S., \& Sarkis, J. (2016). Exploring sub-suppliers' compliance with corporate sustainability standards. Journal of Cleaner Production, 112 (Part3, January), 971-1984. 
[17] Nagalingam, S. V., Kuik, S. S., \& Amer, Y. (2013). Performance measurement of product returns with recovery for sustainable manufacturing. Robotics and Computer-Integrated Manufacturing, 29(6), 473-483.

[18] Treiblmaier, H. (2018). The impact of the blockchain on the supply chain: A theory-based research framework and a call for action. Supply Chain Management: An International Journal, 23(6), 545-559.

[19] Saberi, S., Kouhizadeh, M., Sarkis, J., \& Shen, L. (2019). Blockchain technology and its relationships to sustainable supply chain management. International Journal of Production Research, 57(7), 2117-2135.

[20] Crosby, M., Pattanayak, P., Verma, S., \& Kalyanaraman, V. (2016). Blockchain technology: Beyond bitcoin. Applied Innovation, 2(6-10), 7-19.

[21] Mathiyazhagan, K., Govindan, K., NoorulHaq, A., \& Geng, Y. (2013). An ISM approach for the barrier analysis in implementing green supply chain management. Journal of Cleaner Production, 47 (May), 283297.

[22] Maloni, M. J., \& Brown, M. E. (2006). Corporate social responsibility in the supply chain: An application in the food industry. Journal of Business Ethics, 68(1), 35-52.

[23] Quarshie, A. M., Salmi, A., \& Leuschner, R. (2016). Sustainability and corporate social responsibility in supply chains: The state of research in supply chain management and business ethics journals. Journal of Purchasing and Supply Management, 22(2), 82-97.

[24] Tate, W. L., Ellram, L. M., \& Kirchoff, J. F. (2010). Corporate social responsibility reports: A thematic analysis related to supply chain management. Journal of Supply Chain Management, 46(1), 19-44.

[25] Wang, Y., Singgih, M., Wang, J., \& Rit, M. (2019). Making sense of blockchain technology: How will it transform supply chains?. International Journal of Production Economics, 211(May), 221-236.

[26] Iansiti, M., \& Lakhani, K. R. (2017). The truth about blockchain. Harvard Business Review, 95(1), 118-127.

[27] Nakamoto, S. (2009). Bitcoin open source implementation of P2P currency. Retrieved from https://satoshi.nakamotoinstitute.org/posts/p2pfoundation/1/

[28] Bahga, A., \& Madisetti, V. K. (2016). Blockchain platform for industrial internet of things. Journal of Software Engineering and Applications, 9(10), 533-546

[29] Tapscott, D., \& Tapscott, A. (2017). How blockchain will change organizations. MIT Sloan Management Review, 58(2), 10-13

[30] Yermack, D. (2017). Corporate governance and blockchains. Review of Finance, 21(1), 7-31.

[31] Raval, S. (2016). Decentralized applications: Harnessing Bitcoin's blockchain technology. Sebastopol, CA: O'Reilly Media Inc.

[32] IBM Institute for Business Value. (2015). Device democracy: Saving the future of the Internet of Things. Retrieved from https://www.ibm.com/downloads/cas/Y5ONA8EV

[33] Puthal, D., Malik, N., Mohanty, S. P., Kougianos, E., \& Yang, C. (2018). The blockchain as a decentralized security framework [future directions]. IEEE Consumer Electronics Magazine, 7(2), 18-21.

[34] Zheng, Z., Xie, S., Dai, H., Chen, X., \& Wang, H. (2017, June). An overview of blockchain technology: Architecture, consensus, and future trends. In G. Karypis \& J Zhang (Eds.), 2017 IEEE $6^{\text {th }}$ International Congress on Big Data (pp. 557-564). https://doi.org/10.1109/BigDataCongress.2017.85 
[35] Xiong, Z., Zhang, Y., Niyato, D., Wang, P., \& Han, Z. (2018). When mobile blockchain meets edge computing. IEEE Communications Magazine, 56(8), 33-39.

[36] Watanabe, H., Fujimura, S., Nakadaira, A., Miyazaki, Y., Akutsu, A., \& Kishigami, J. J. (2015, October). Blockchain contract: A complete consensus using blockchain. In 2015 IEEE 4th global conference on $\begin{array}{lllll}\text { consumer } & \text { electronics } & \text { (GCCE), } & \text { R77-578. }\end{array}$ https://www.researchgate.net/profile/Hiroki_Watanabe17/publication/304298870_Blockchain_contract_A complete_consensus_using_blockchain/links/5b51bffd45851507a7b282e7/Blockchain-contract-Acomplete-consensus-using-blockchain.pdf

[37] Tian, F. (2016, June). An agri-food supply chain traceability system for China based on RFID \& blockchain technology. In 2016 13th international conference on service systems and service management (ICSSSM) (pp. 1-6). IEEE. Retrieved from https://epub.wu.ac.at/6090/1/Dissertation_of_Feng_Tian.pdf\#page=66

[38] Li, Y., Wang, B., \& Yang, D. (2019). Research on Supply Chain Coordination Based on Block Chain Technology and Customer Random Demand. Discrete Dynamics in Nature and Society, Advance online publication. https://doi.org/10.1155/2019/4769870

[39] Pfeffer, J. (2010). Building sustainable organizations: The human factor. Academy of Management Perspectives, 24(1), 34-45.

[40] Egels-Zandén, N. (2014). Revisiting supplier compliance with MNC codes of conduct: Recoupling policy and practice at Chinese toy suppliers. Journal of Business Ethics, 119(1), 59-75.

[41] Welford, R., \& Frost, S. (2006). Corporate social responsibility in Asian supply chains. Corporate Social Responsibility and Environmental Management, 13(3), 166-176.

[42] Ciliberti, F., de Groot, G., de Haan, J., \& Pontrandolfo, P. (2009). Codes to coordinate supply chains: SMEs' experiences with SA8000. Supply Chain Management: An International Journal, 14(2), 117-127.

[43] Zakaria, M., Garanča, Z., \& Sobeih, A. (2012). Cultural and legal challenges in implementing code of conduct in supply chain management of mobile phone industries: Sony Ericsson case study. Social Responsibility Journal, 8(2), 227-241.

[44] Blome, C., \& Paulraj, A. (2013). Ethical climate and purchasing social responsibility: A benevolence focus. Journal of Business Ethics, 116(3), 567-585.

[45] Mamic, I. (2005). Managing global supply chain: The sports footwear, apparel and retail sectors. Journal of Business Ethics, 59(1-2), 81-100.

[46] Barrientos, S., \& Smith, S. (2007). Do workers benefit from ethical trade? Assessing codes of labor practice in global production systems. Third World Quarterly, 28(4), 713-729.

[47] Egels-Zandén, N., \& Merk, J. (2014). Private regulation and trade union rights: Why codes of conduct have limited impact on trade union rights. Journal of Business Ethics, 123(3), 461-473.

[48] Hale, A., \& Wills, J. (2007). Women working worldwide: Transnational networks, corporate social responsibility and action research. Global Networks, 7(4), 453-476.

[49] Ruwanpura, K. N., \& Wrigley, N. (2011). The costs of compliance? Views of Sri Lankan apparel manufacturers in times of global economic crisis. Journal of Economic Geography, 11(6), 1031-1049.

[50] Perry, P., Wood, S., \& Fernie, J. (2015). Corporate social responsibility in garment sourcing networks: Factory management perspectives on ethical trade in Sri Lanka. Journal of Business Ethics, 130(3), 737-752.

[51] Wilhelm, M. M., Blome, C., Bhakoo, V., \& Paulraj, A. (2016). Sustainability in multi-tier supply chains: Understanding the double agency role of the first-tier supplier. Journal of Operations Management, 41 (January), 42-60.

[52] Tachizawa, E. M., \& Yew Wong, C. (2014). Towards a theory of multi-tier sustainable supply chains: A systematic literature review. Supply Chain Management: An International Journal, 19(5/6), 643-663. 
[53] Mahmood, I. P., Zhu, H., \& Zajac, E. J. (2011). Where can capabilities come from? Network ties and capability acquisition in business groups. Strategic Management Journal, 32(8), 820-848.

[54] Petersen, K. J., Handfield, R. B., \& Ragatz, G. L. (2005). Supplier integration into new product development: Coordinating product, process and supply chain design. Journal of Operations Management, 23(3-4), 371388 .

[55] Nikolakis, W., John, L., \& Krishnan, H. (2018). How blockchain can shape sustainable global value chains: An evidence, verifiability, and enforceability (EVE) framework. Sustainability, 10(11), 1-16

[56] Kong, X. T., Yang, X., Huang, G. Q., \& Luo, H. (2018). The impact of industrial wearable system on industry 4.0. In 2018 IEEE 15th International Conference on Networking, Sensing and Control (ICNSC), 1-6. IEEE. https//doi.org/10.1109/ICNSC.2018.8361266

[57] Zhong, R. Y., Huang, G. Q., Lan, S., Dai, Q. Y., Chen, X., \& Zhang, T. (2015). A big data approach for logistics trajectory discovery from RFID-enabled production data. International Journal of Production Economics, 165 (July), 260-272.

[58] Panarello, A., Tapas, N., Merlino, G., Longo, F., \& Puliafito, A. (2018). Blockchain and IoT integration: A systematic survey. Sensors, 18(8), 2575.

[59] Schmidt, G., \& Wilhelm, W. E. (2000). Strategic, tactical and operational decisions in multi-national logistics networks: A review and discussion of modelling issues. International Journal of Production Research, 38(7), 1501-1523.

[60] Zhong, R. Y., Huang, G. Q., Dai, Q. Y., \& Zhang, T. (2014). Mining sots and dispatching rules from RFIDenabled real-time shop floor production data. Journal of Intelligent Manufacturing, 25(4), 825-843.

[61] Zhang, Y., Jiang, P., Huang, G., Qu, T., Zhou, G., \& Hong, J. (2012). RFID-enabled real-time manufacturing information tracking infrastructure for extended enterprises. Journal of Intelligent Manufacturing, 23(6), 2357-2366.

[62] Francisco, K., \& Swanson, D. (2018). The supply chain has no clothes: Technology adoption of blockchain for supply chain transparency. Logistics, 2(1), 2.

[63] Kouhizadeh, M., \& Sarkis, J. (2018). Blockchain practices, potentials, and perspectives in greening supply chains. Sustainability, 10(10), 3652.

[64] Mezzadri, A. (2014). Indian garment clusters and CSR norms: Incompatible agendas at the bottom of the garment commodity chain. Oxford Development Studies, 42(2), 238-258.

[65] Hutchins, M. J., \& Sutherland, J. W. (2008). An exploration of measures of social sustainability and their application to supply chain decisions. Journal of Cleaner Production, 16(15), 1688-1698.

[66] Haas, E. J., \& Yorio, P. (2016). Exploring the state of health and safety management system performance measurement in mining organizations. Safety Science, 83 (March), 48-58.

[67] Huang, G. Q., Qu, T., Fang, M. J., \& Bramley, A. N. (2011). RFID-enabled gateway product service system for collaborative manufacturing alliances. CIRP Annals - Manufacturing Technology, 60(1), 465-468.

[68] Roy, R. (2000). Sustainable product-service systems. Futures, 32(3), 289-299.

[69] A software engineering salary survey. Retrieved March 23, 2019, from https://www.job592.com/direction/job100008/. 
[70] Chen, T., \& Tsai, H. R. (2017). Ubiquitous manufacturing: Current practices, challenges, and opportunities. Robotics and Computer-Integrated Manufacturing, 45(June), 126-132.

[71] Qu, T., Chen, X. D., Zhang, Y., Yang, H., \& Huang, G. Q. (2011). Analytical target cascading-enabled optimal configuration platform for production service systems. International Journal of Computer Integrated Manufacturing, 24(5), 457-470.

[72] Tukker, A., \& Tischner, U. (2006). Product-services as a research field: Past, present and future. Reflections from a decade of research. Journal of Cleaner Production, 14(17), 1552-1556.

[73] Yuan, Y., \& Wang, F. Y. (2018). Blockchain and cryptocurrencies: Model, techniques, and applications. IEEE Transactions on Systems, Man, and Cybernetics: Systems, 48(9), 1421-1428.

[74] Wang, Y., Han, J. H., \& Beynon-Davies, P. (2019). Understanding blockchain technology for future supply chains: A systematic literature review and research agenda. Supply Chain Management: An International Journal, 24(1), 62-84.

[75] Hofman, D., Lemieux, V. L., Joo, A., \& Batista, D. A. (2019). "The margin between the edge of the world and infinite possibility" Blockchain, GDPR and information governance. Records Management Journal, 29(1/2), 240-257.

[76] Tarafdar, M., Gupta, A., \& Turel, O. (2013). The dark side of information technology use. Information Systems Journal, 23(3), 269-275.

[77] Hull, R. (2017, June). Blockchain: Distributed event-based processing in a data-centric world. In Proceedings of the 11th ACM International Conference on Distributed and Event-based Systems (pp. 2-4). ACM.3 doi \# $10.1145 / 3093742.3097982$

[78] Patel, D., Bothra, J., \& Patel, V. (2017, January). Blockchain exhumed. In 2017 ISEA Asia Security and Privacy (ISEASP) (pp. 1-12). IEEE. https;//doi.org/10.13140/RG.2.2.35506.45765

[79] Chenhall, R. H., Hall, M., \& Smith, D. (2010). Social capital and management control systems: A study of a non-government organization. Accounting, Organizations and Society, 35(8), 737-756.

[80] Fu, B., Shu, Z., \& Liu, X. (2018). Blockchain enhanced emission trading framework in fashion apparel manufacturing industry. Sustainability, 10(4), 1105-1124

[81] Zhang, A., Luo, H., Huang, G. Q. (2013). A bi-objective model for supply chain design of dispersed manufacturing in China. International Journal of Production Economics, 146(1), 48-58. 